See discussions, stats, and author profiles for this publication at: https://www.researchgate.net/publication/264417628

\title{
LA DIVERSITE INVISIBLE: AU-DELÀ DE CE QUE L'ON PEUT VOIR
}

\author{
Book · July 2014 \\ DOI: 10.13140/2.1.5087.0403
}

3 authors:

51 PUBLICATIONS 73 CITATIONS 55 PUBLICATIONS 227 CITATIONS

SEE PROFILE

SEE PROFILE

Marcelo Pessoa de Oliveira

Universidade do Estado de Minas Gerais

9 PUBLICATIONS O CITATIONS

SEE PROFILE

Some of the authors of this publication are also working on these related projects:

Core virome characterization of an endangered coral species in southwest, Puerto Rico View project

A description of a new genus of Parastenocaridinae from Brazil. With phylogeny of the subfamily Parastenocaridinae. View project 


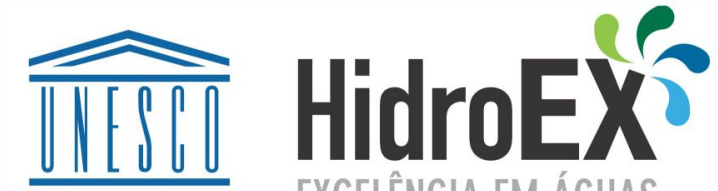 \\ EXCELÊNCIA EM ÁGUAS}

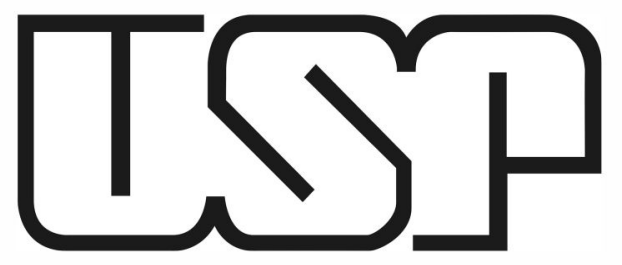

Universidade de São Paulo
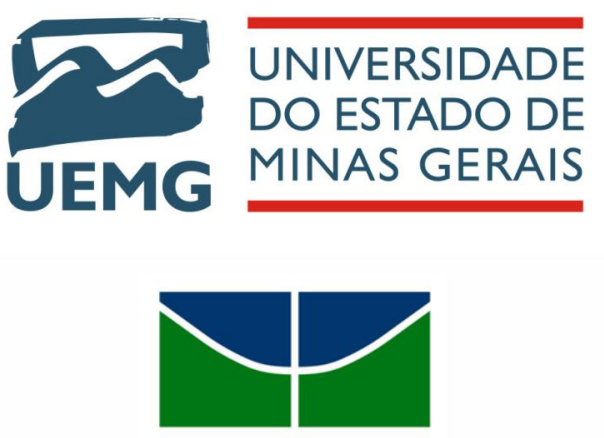

\section{UnB}

Universidade

Católica de Brasília

Agência Brasileira do ISBN

ISBN 978-85-914769-2-3

||||||||||||||||||| |||||||||||||||||||||||||||||||

$9\left\|\prod_{788591}\right\| \|_{476923}$
(-) BIODIVERSITÉ

\section{LA DIVERSITÉ INVISIBLE}

AUU-DPLA DE CE QUE LON PEUT VOIR

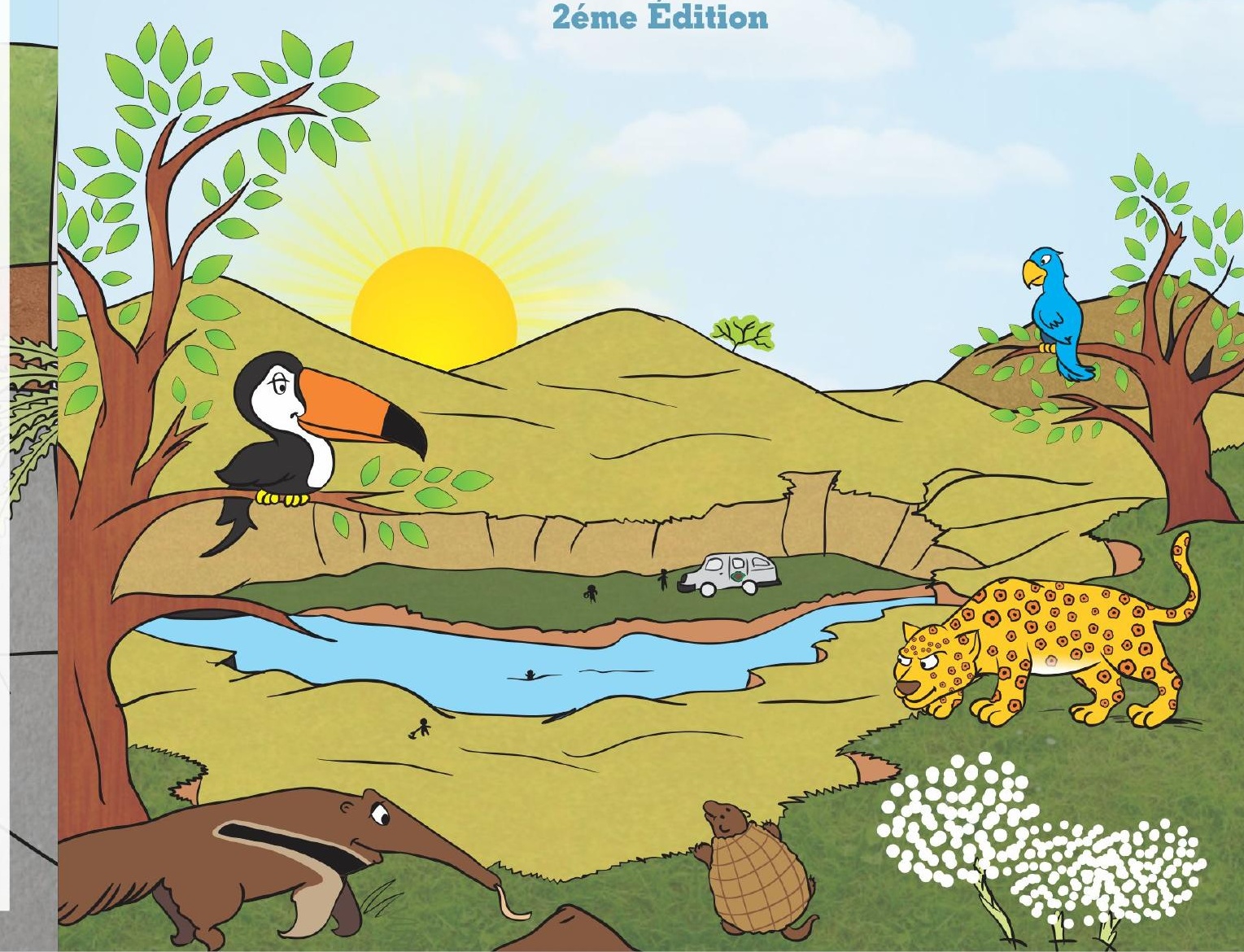




\author{
Daniel Previattelli \\ Marcelo Pessoa \\ Paulo Corgosinho \\ LA DIVERSITÉ \\ INVISIBLE
}

AU-DELÀ DE CE QUE L'ON PEUT VOIR

FICHE CATALOGRAPHIQUE ÉLABORÉE PAR LE SERVICE DE LA BIBLIOTHĖQUE DE L'INSTITUT DES BIOSCIENCES / USP

P944 Previattelli, Daniel

La Diversité invisible: au-delà de ce que l'on peut voir /

Daniel Previattelli, Marcelo Pessoa, Paulo Corgosinho. -- 2. éd. -- São Paulo: CNPQ/FAPESP, 2014.

28p. : il. ; 21 cm. -- (Biodiversité: microcrustacés d'eau douce dans les champs rocheux)

ISBN: 978-85-914769-2-3

1. Crustáceos. 2. Biodiversidade. I. Pessoa, Marcelo. II Corgosinho, Paulo. III. Título. 
Édition

Dr. Daniel Previattelli

\section{Paternité}

Dr. Daniel Previattelli

Dr. Marcelo Pessoa

Dr. Paulo Henrique Costa Corgosinho

\section{Ilustration et composition}

Victor Machado Martins

\section{Création du logotype}

Diego David dos Santos Silva

\section{Traduction}

Ana Brancelj

\section{Lecture et correction}

Ana Brancelj

Cristiane Freitas de Azevedo Barros
Le mot "rocheux" se rapporte aux rochers. C'est pourquoi un champs rocheux c'est ou nous voyons beaucoup de pierres, ainsi que les broussailles et très peud'arbres aux troncs noueux.

Dans ces environnements il y a une grande biodiversité: les différents animaux tels que le loup à crinière, le tamanoir et le cariama, ainsi que les plantes très caractéristiques comme par exemple la pâquerette et vellozia, une sorte d'iris. L'eau est d'une grande importance dans ces champs. II y a dedans, a part des poissons, aussi une biodiversité invisible. Ce sont ainsi dit microcrustacés et les micro-algues, qui, elles aussi contribuent à l'équilibre de ces écosystèmes.

Les microcrustacés sont parfois donnés le nom de puces d'eau, dû au fait qu'ils nagent très rapidement, faisant des mouvements brusques qui rappellent aux sauts de puces.

Ce monde invisible et tout qui s'y passe est tellement fascinant, qu'ily existe une science particulière qui s'en occupe, et qui attire des centaines des chercheurs du monde entier.

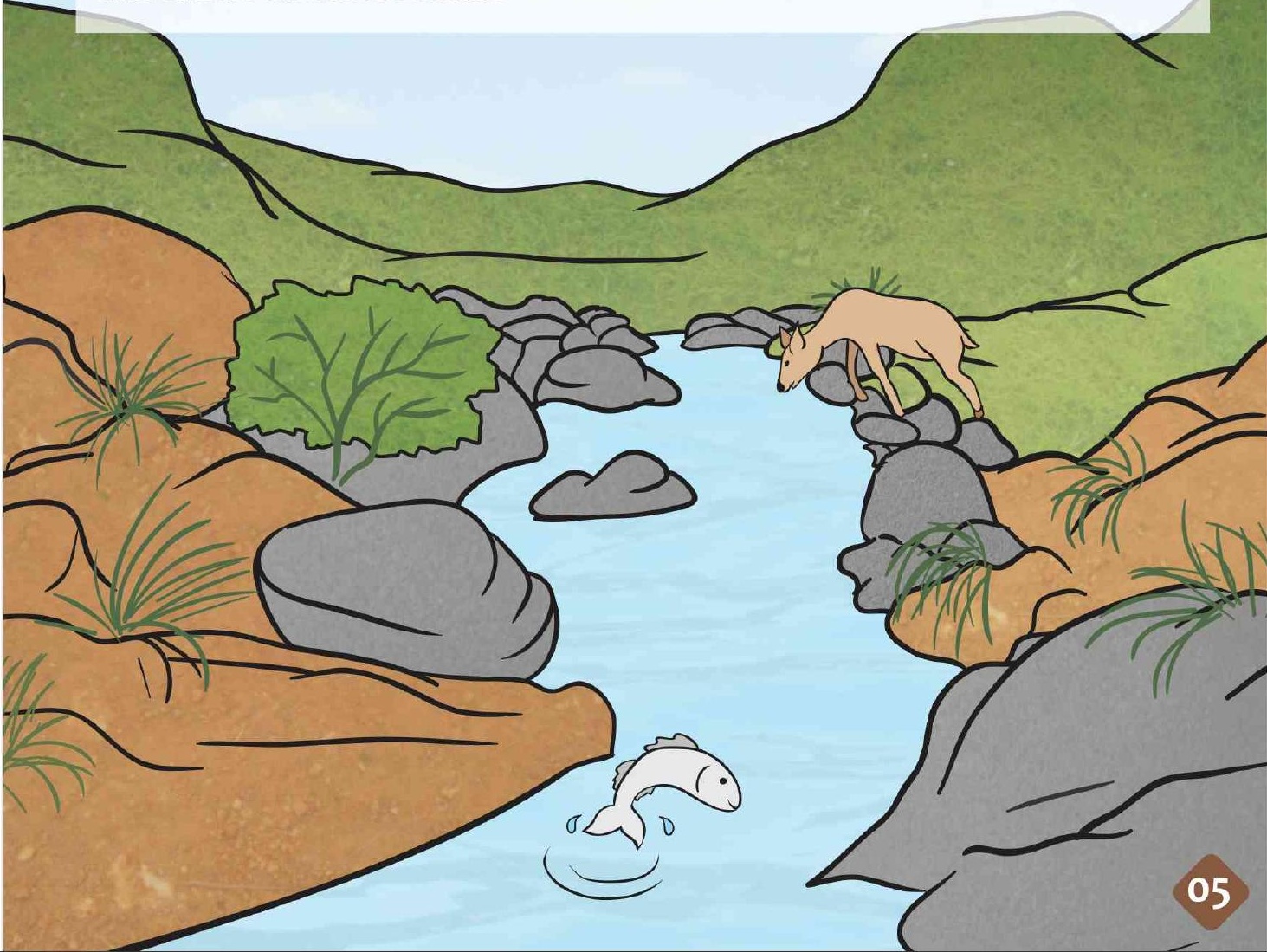


Coucou, je suis Cyclopotche, mais tu peux m'appeler la "crevette invisible" ou puce d'eau! Mais ne t'inquiètes pas! Je ne vais pas te démanger ni te faire du mal.

Tu vois, un champ rocheux ne contient pas que les pierres et les arbres tortueux. II y a des plantes invisibles comme par exemple les micro-algues et les animaux minuscules comme moi, appelés microcrustacés. Oh lá lá, une famille difficile à comprendre ! Quand on les observe par un microscope, ils ressemblent aux petites crevettes, aux minimes araignées ou parfois aux petits scarabées.

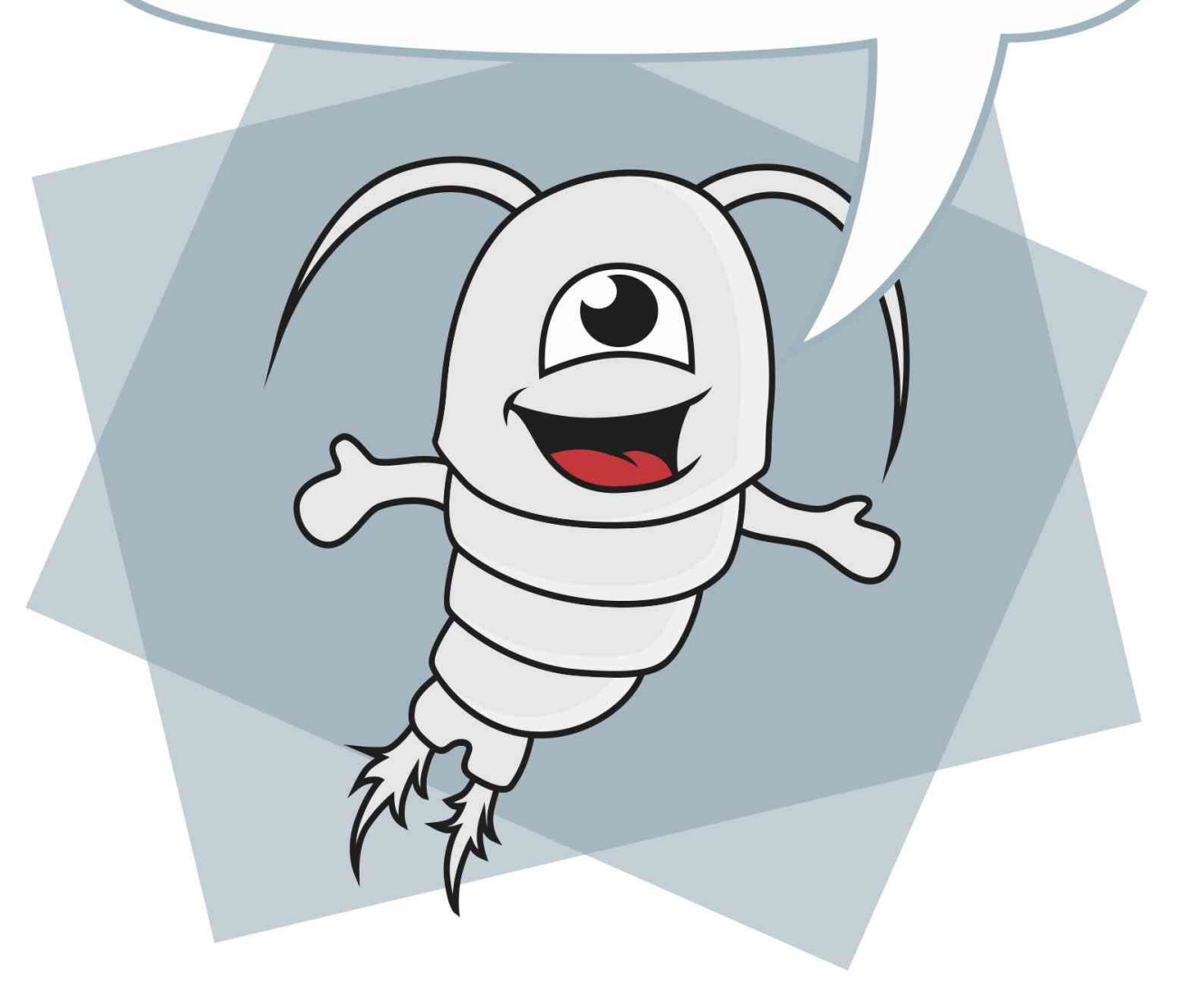

\section{MICRO - MAGRO}

Quand tu regardes l'eau tellement cristalline d'un fleuve, tu ne t'imagines pas qu'il y existe une beauté au-delà de ce que tes yeux peuvent percevoir.

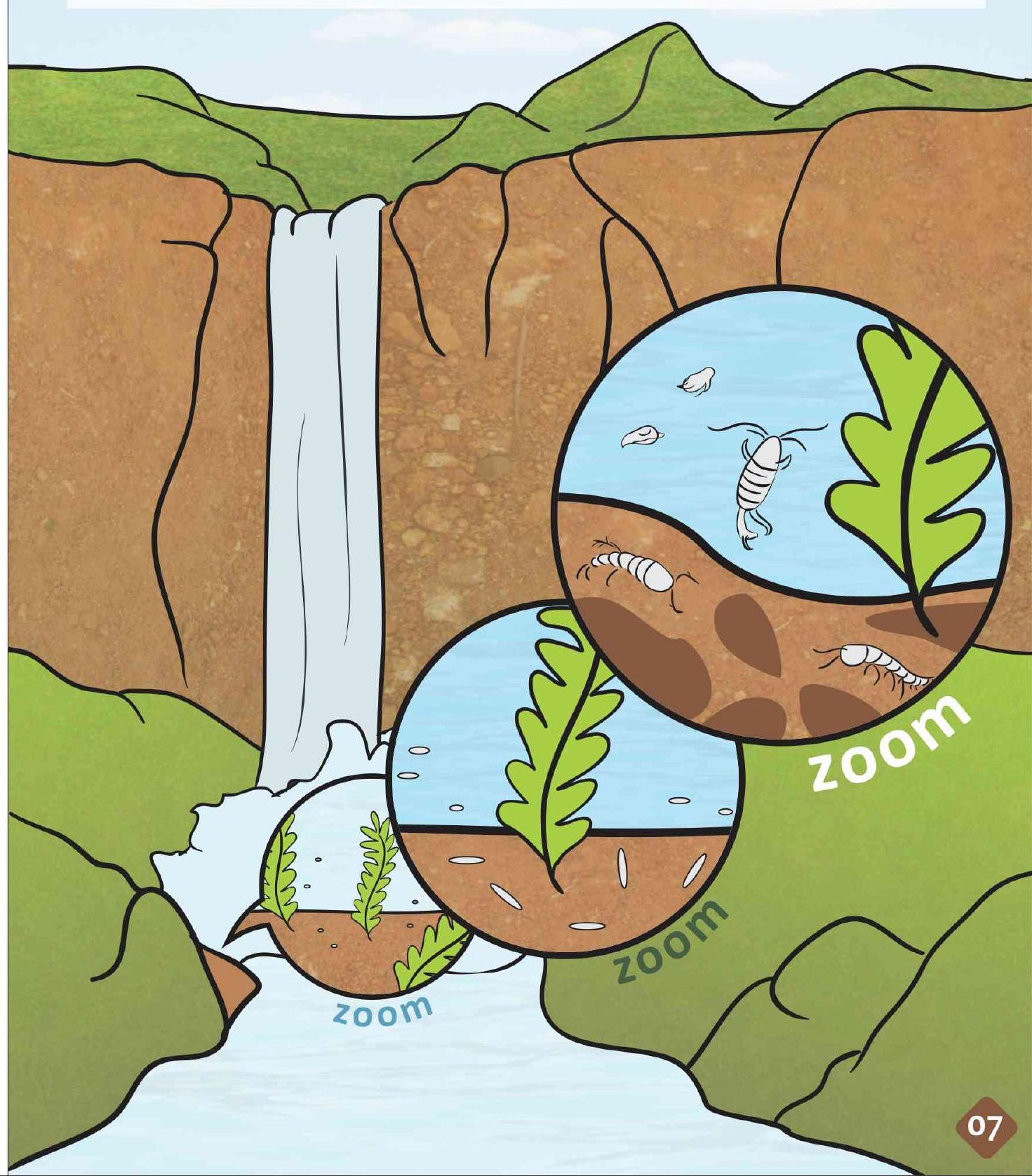




\section{CHIST NOUS}

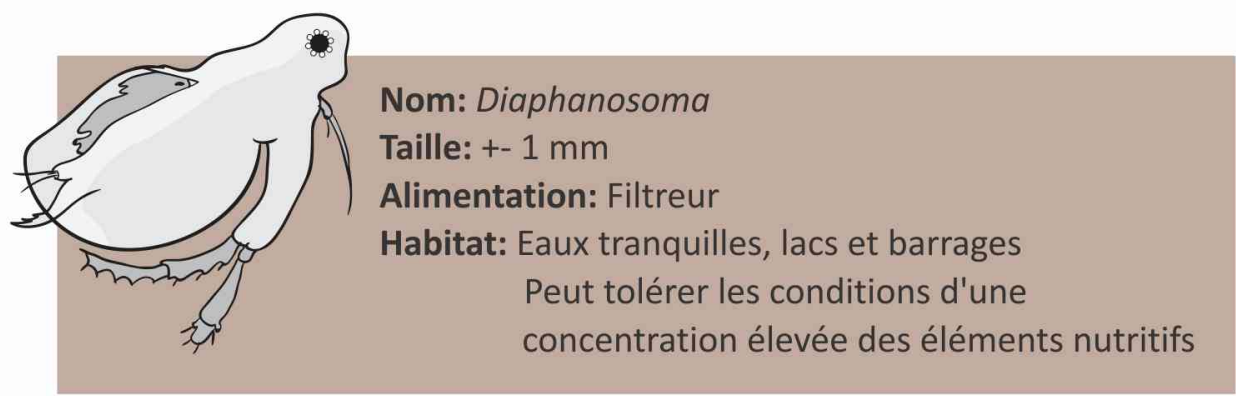

Nom: Chydoridae

Taille: De 0,3 à 0,5 mm

Alimentation: Gratteurs des micro-algues

Habitat: Agrippés au sol, peuvent se trouver aussi

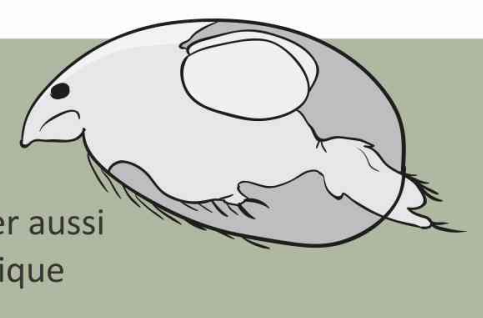

attachés à la végétation subaquatique

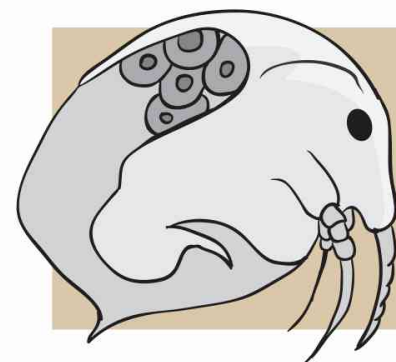

Nom: Bosmina

Taille: Moins que $0,5 \mathrm{~mm}$

Alimentation: Filtreur

Habitat: Se présentent dans les eaux où la

concentration des élément nutritifs est plus basse

Nom: Parastenocarididae

Taille: De 0,3 à $0,4 \mathrm{~mm}$

Alimentation: Détritivores ou gratteurs

Habitat: Attachés au sol humide ou mouillé des fonds des fleuves, des lacs et de leurs bords. Peuvent se présenter dans les eaux d'une basse concentration des éléments nutritifs

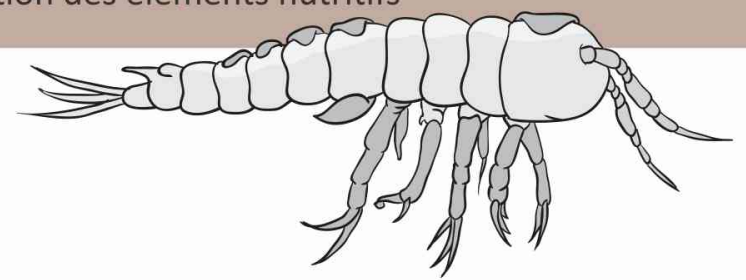

Nom: Cyclopoida

Taille: De 0,4 à $1 \mathrm{~mm}$ et plus

Alimentation: Prédateurs, détritivores, gratteurs

Habitat: Nagent librement dans l'eau ou peuvent être attachés au sol ainsi qu'à la végétation immergée. Se trouvent dans les différents types de milieux de différentes concentrations des éléments nutritifs

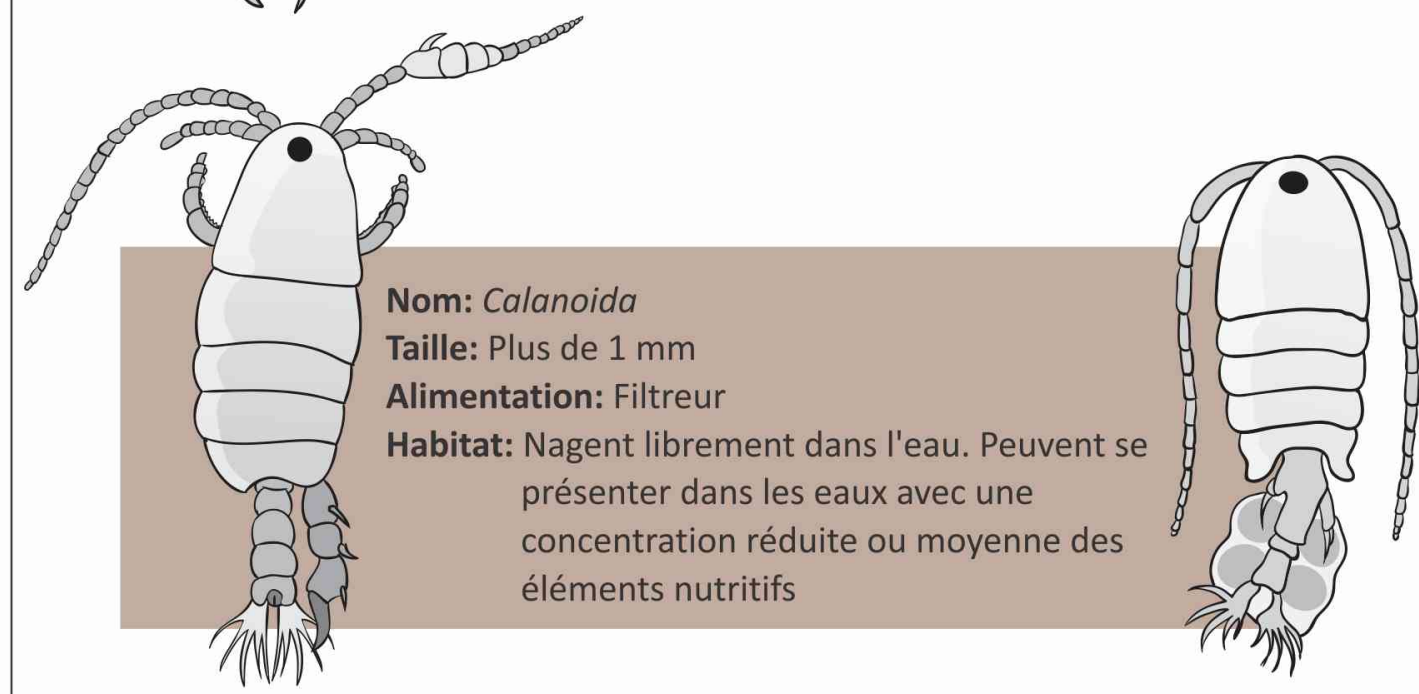

\section{Nom: Canthocamptidae}

Taille: De 0,5 à 0,8 mm

Alimentation: Détritivore ou gratteur

Habitat: Attachés aux sols humides ou mouillés des fonds des fleuves, des lacs et de leurs bords. Peuvent se présenter dans les eaux d'une concentration basse ou moyenne des éléments nutritifs 


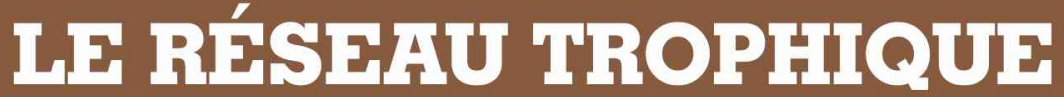
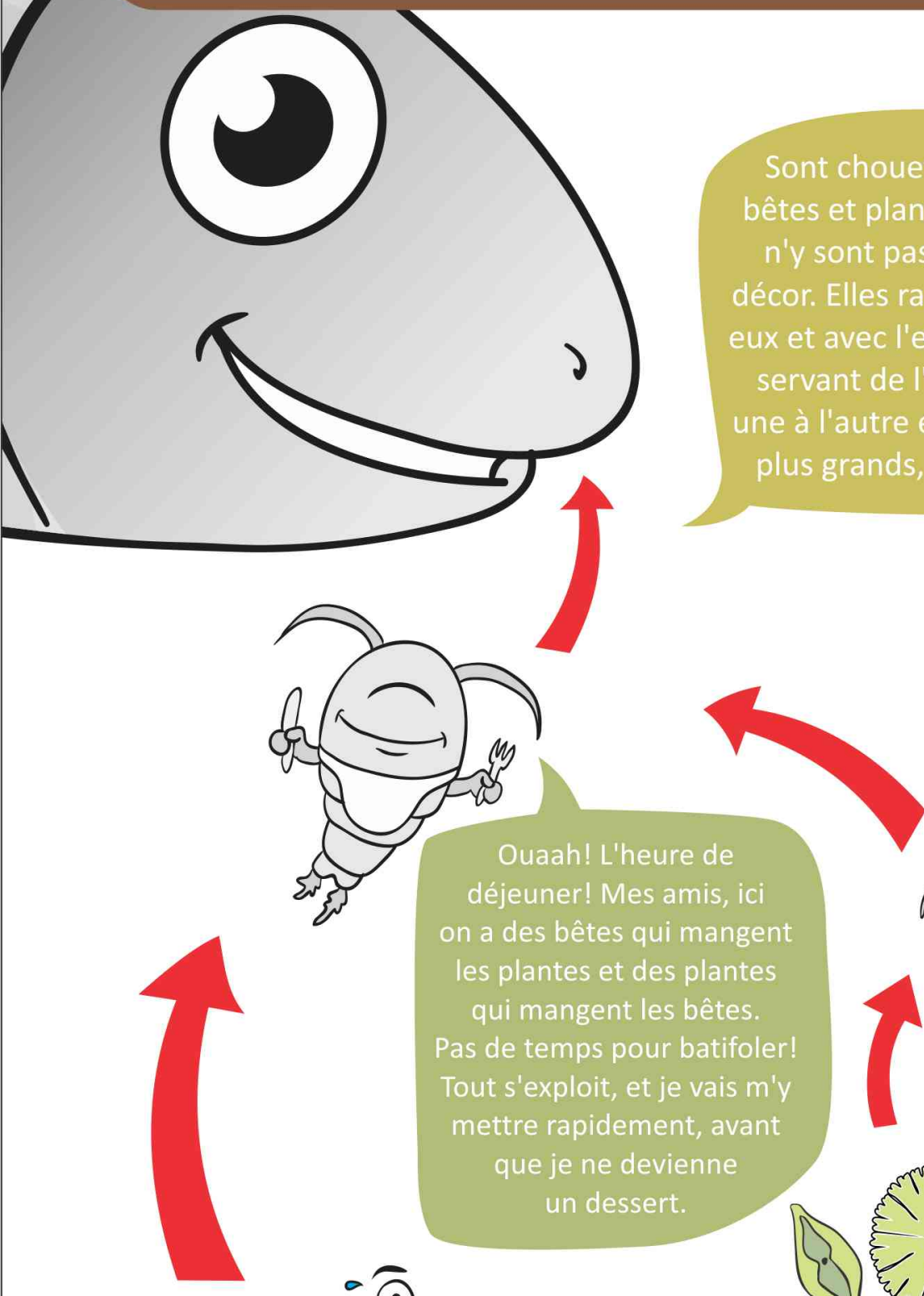

bêtes et plantes, mais elles

n'y sont pas que pour le

décor. Elles raccordent entre

eux et avec l'environnement,

servant de l'alimentation

une à l'autre et aux animaux

plus grands, comme moi.
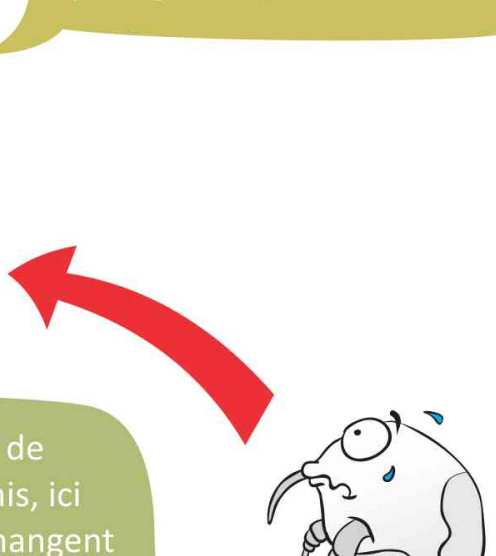

on a des bêtes qui mangent

les plantes et des plantes

qui mangent les bêtes.

Tout s'exploit, et je vais m'y

mettre rapidement, avant

que je ne devienne

un dessert.
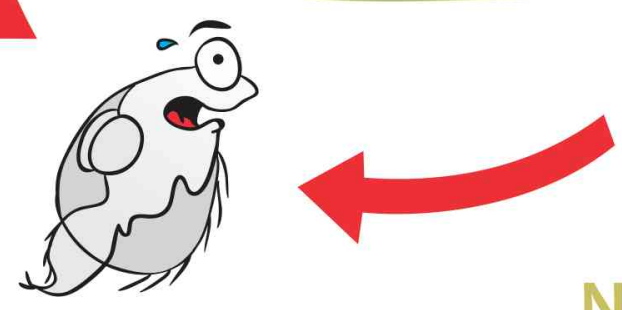

NUTRIMENTS

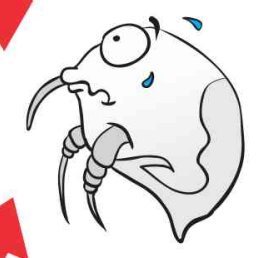

Pas de temps pour batifoler!

\section{LFS FNVIRONMINTS NATURHLS}

Les milieux naturels possèdent une diversité riche d'organismes et de relations écologiques. Quand ils sont bien préservés, ils peuvent nous fournir des

biens importants.
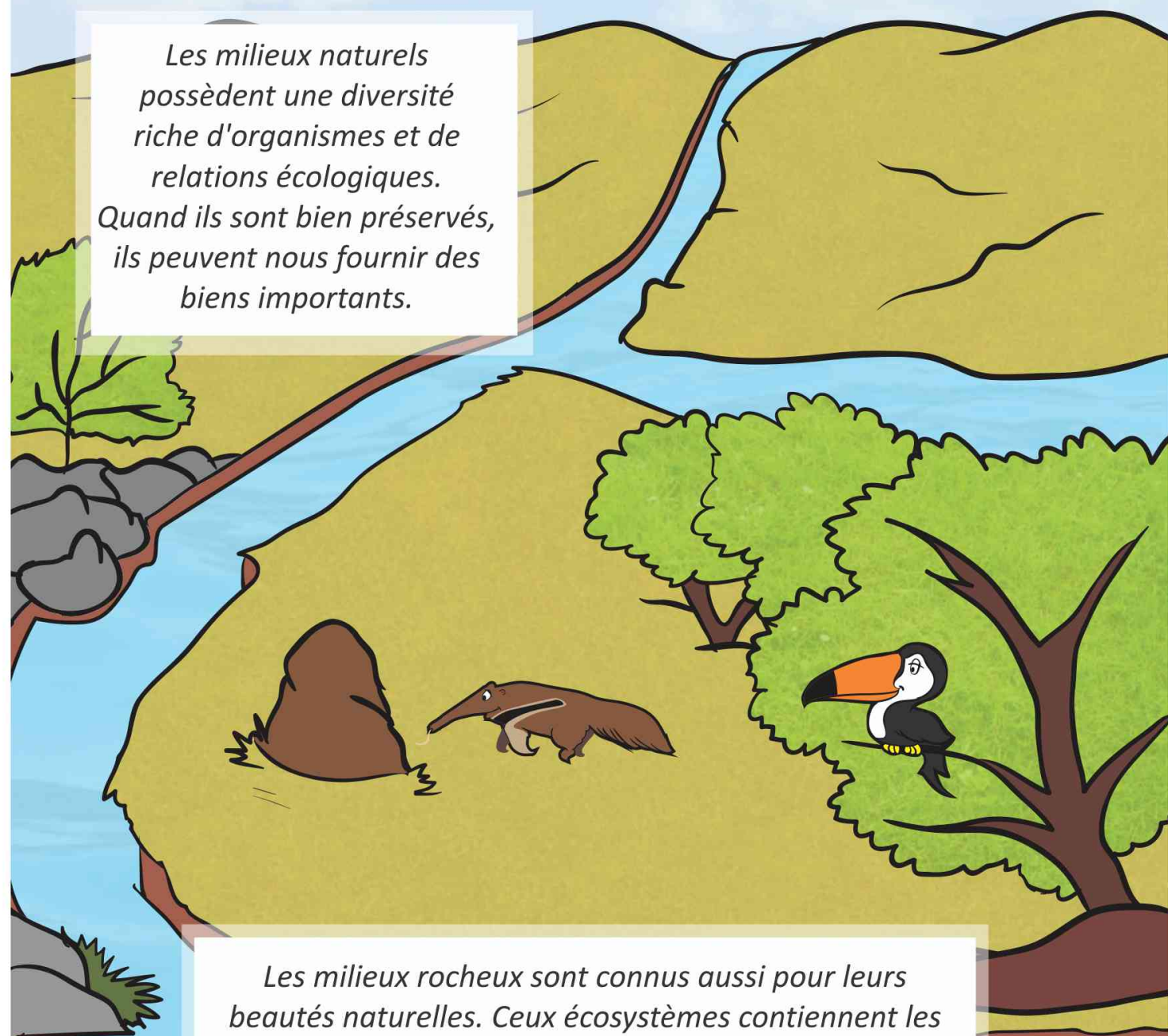

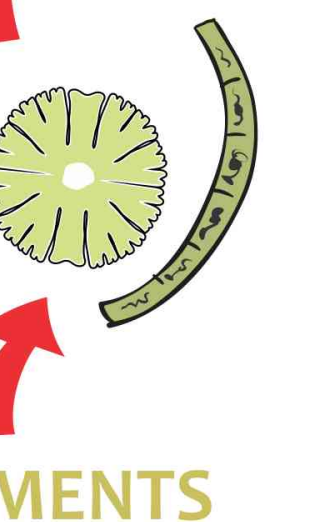

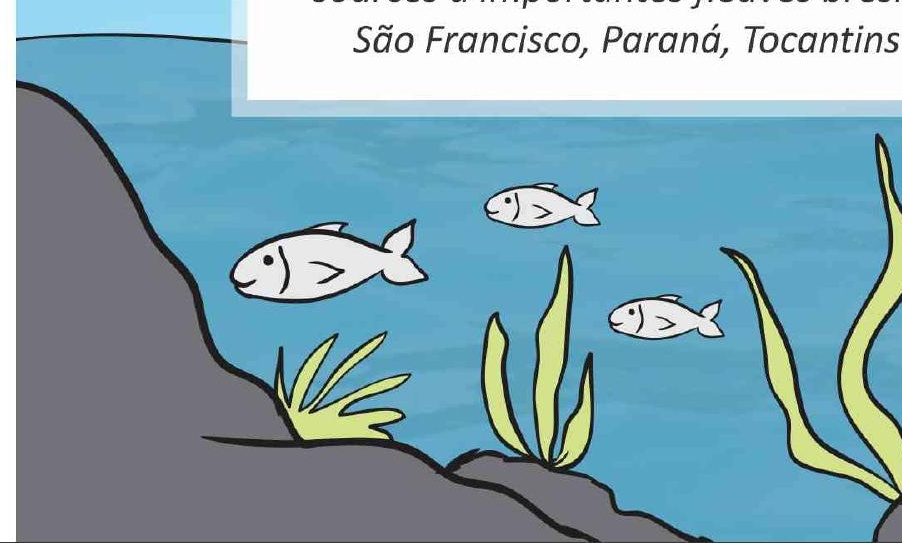

Les milieux rocheux sont connus aussi pour leurs beautés naturelles. Ceux écosystèmes contiennent les sources d'importantes fleuves brésiliennes, comme São Francisco, Paraná, Tocantins et les autres. 
Ouf! Le voyage n'était pas difficile juste parce que on est allé en voiture. Marcher fatigue aussi! Et tout cela pour voir ma famille. Ouah!

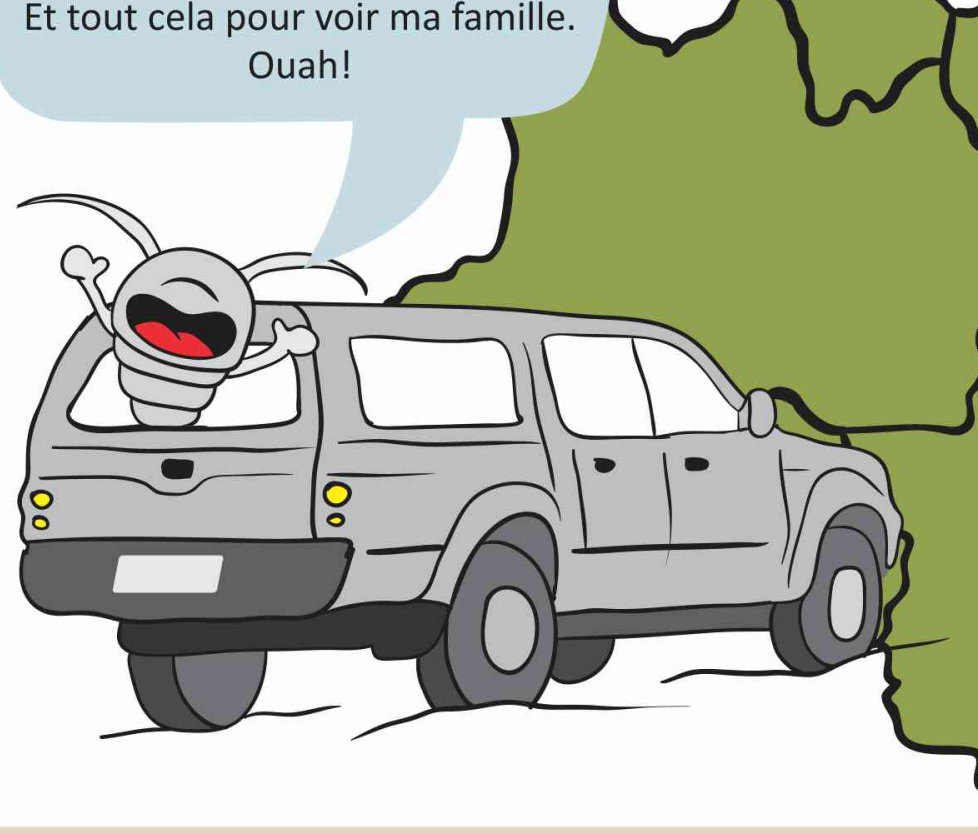

La liste des lieux visités:

1 - Plateau Chapada dos Guimarães, l'état de Mato Grosso

2 - Chaîne de montagnes Serra dos Pirineus- Pirenópolis, l'état de Goiás

3 - Chaîne de montagnes Serra de Caldas Novas, l'état de Goiás

4 - Plateau Chapada dos Veadeiros, l'état de Goiás

5 - Chaîne de montagnes Serra de Cristalina, l'état de Goiás

6 - Chaîne de montagnes Serra da Canastra, l'état de Minas Gerais

7 - Parque d'état Serra do Cabral, l'état de Minas Gerais

8 - Chaîne de montagnes Serra do Cipó, l'état de Minas Gerais

9 - Parque d'état Rio Preto, l'état de Minas Gerais

La collecte est réalisée dans les différents types d'eau: les lacs, étangs, rivières, sources, grottes, cascades, camps secs et humides.

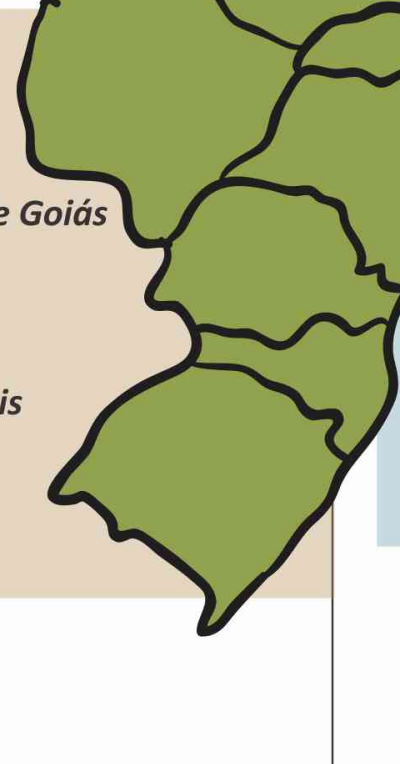

Chaque lieu est visite dans deux saisons: sèche et humide. Ainsi comprenons-nous mieux comment les microcrustacés vivent dans les différentes conditions. Au total, l'équipe a parcouru au cours du projet, plus de 250 mille kilomètres.

L'équipe fait face aux lieux de collecte parfois très secs, parfois trompés, ou même submergés par les inondations temporelles.
Les chercheurs affrontent les températures qui Les chercheurs affrontent les températures qui
varient d'environ $45^{\circ} \mathrm{C}$ en été, jusqu'à $2^{\circ} \mathrm{C}$ en hiver.

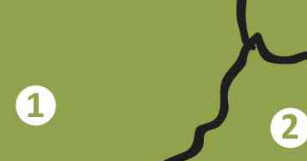

(1) (n)

$2^{6}$

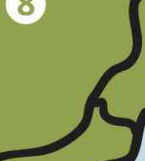

Une grande partie de chemin, jusqu'aux points de collecte, est faite à pied. Une équipe de 4 personnes fait les expéditions qui varient entre 100 et 8000 mètres de distance. En plus, l'équipe transport environ 200 kilos de matériel de laboratoire, de microcrustacés ramassés et de cameras. 


\section{LAS ÉTAPES DE RECHERCHE TH COTMJCHH}

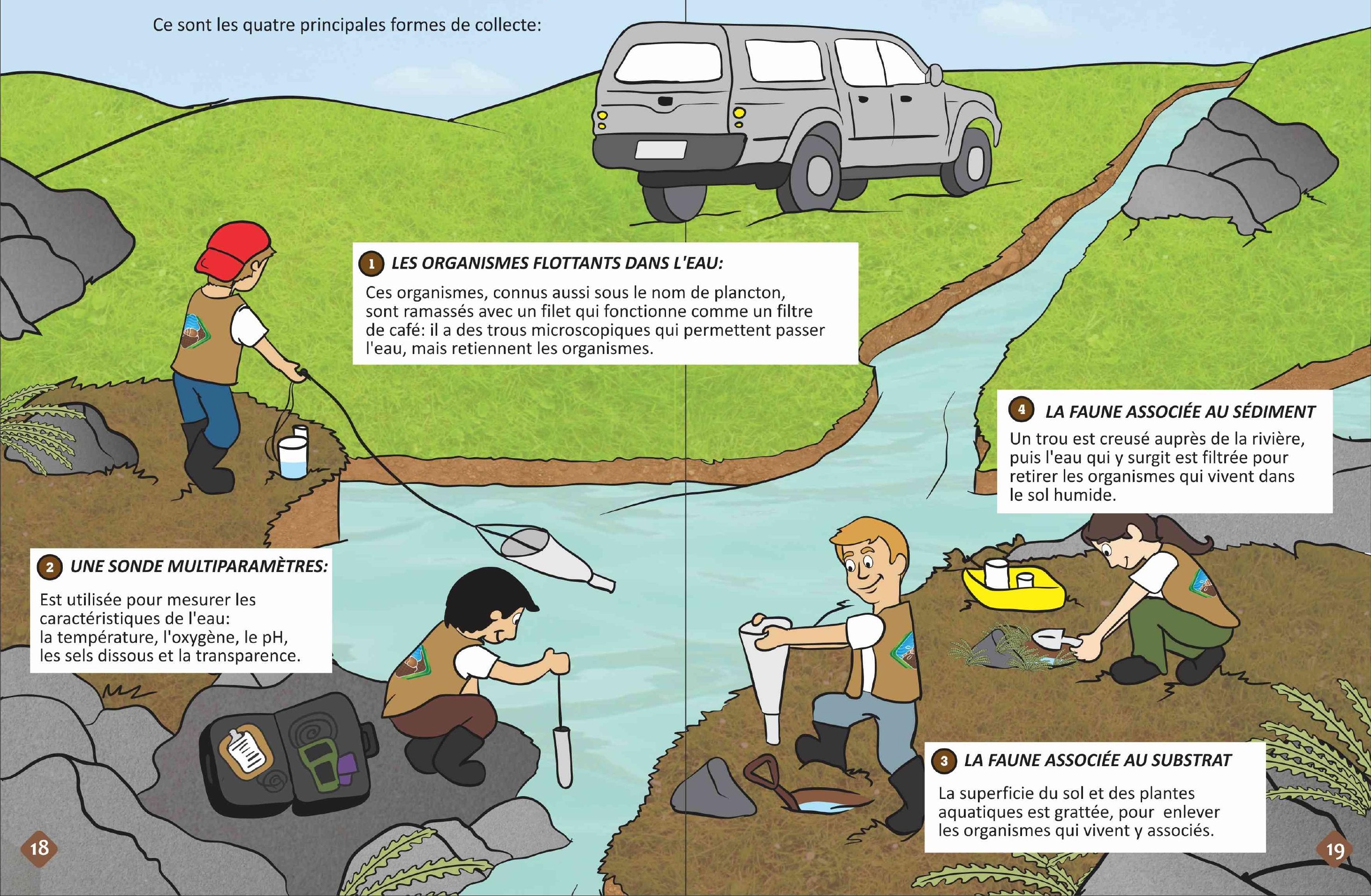




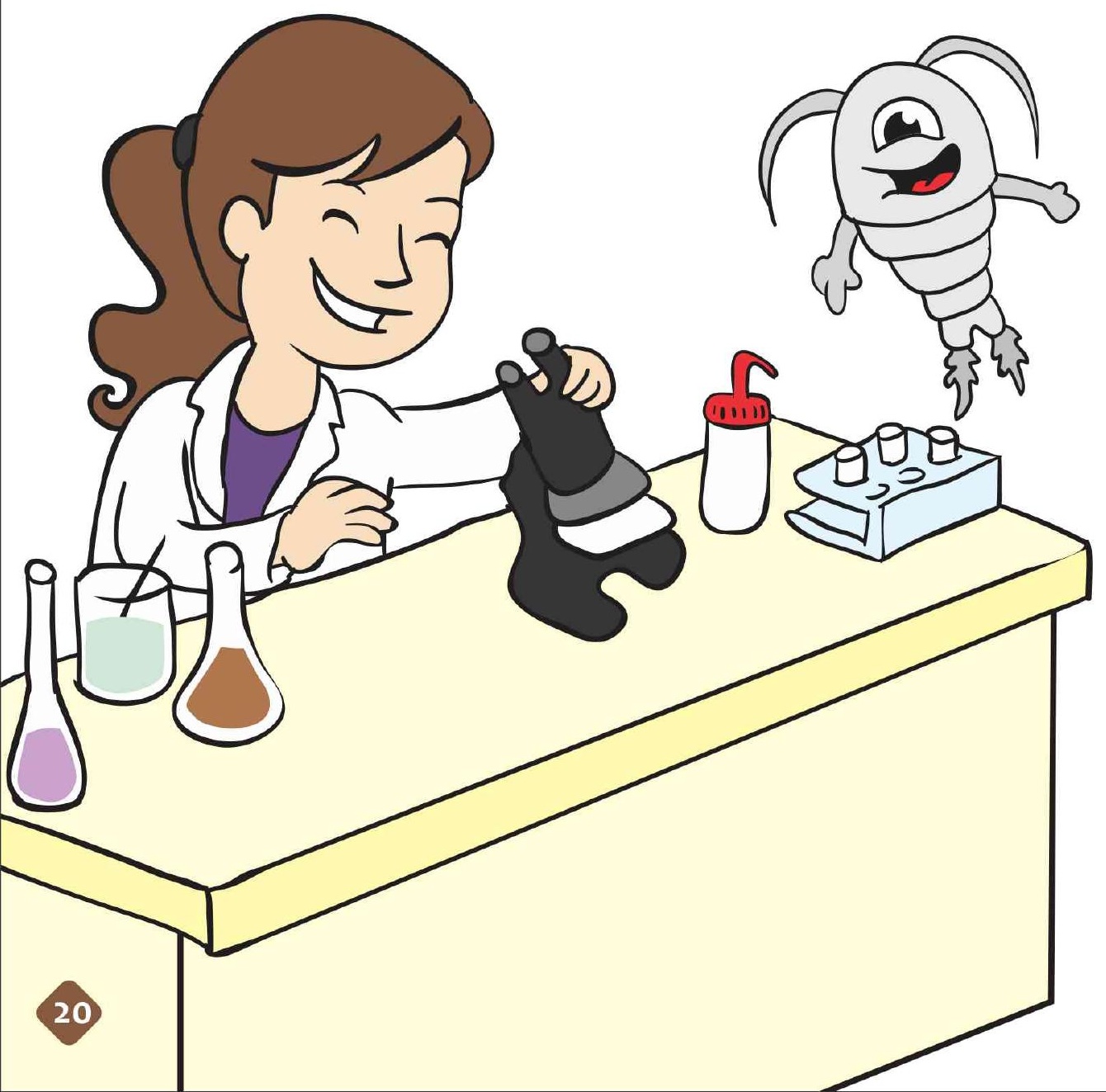

Regarde! Le chercheur nous scrute à la loupe! Ha ha ha!

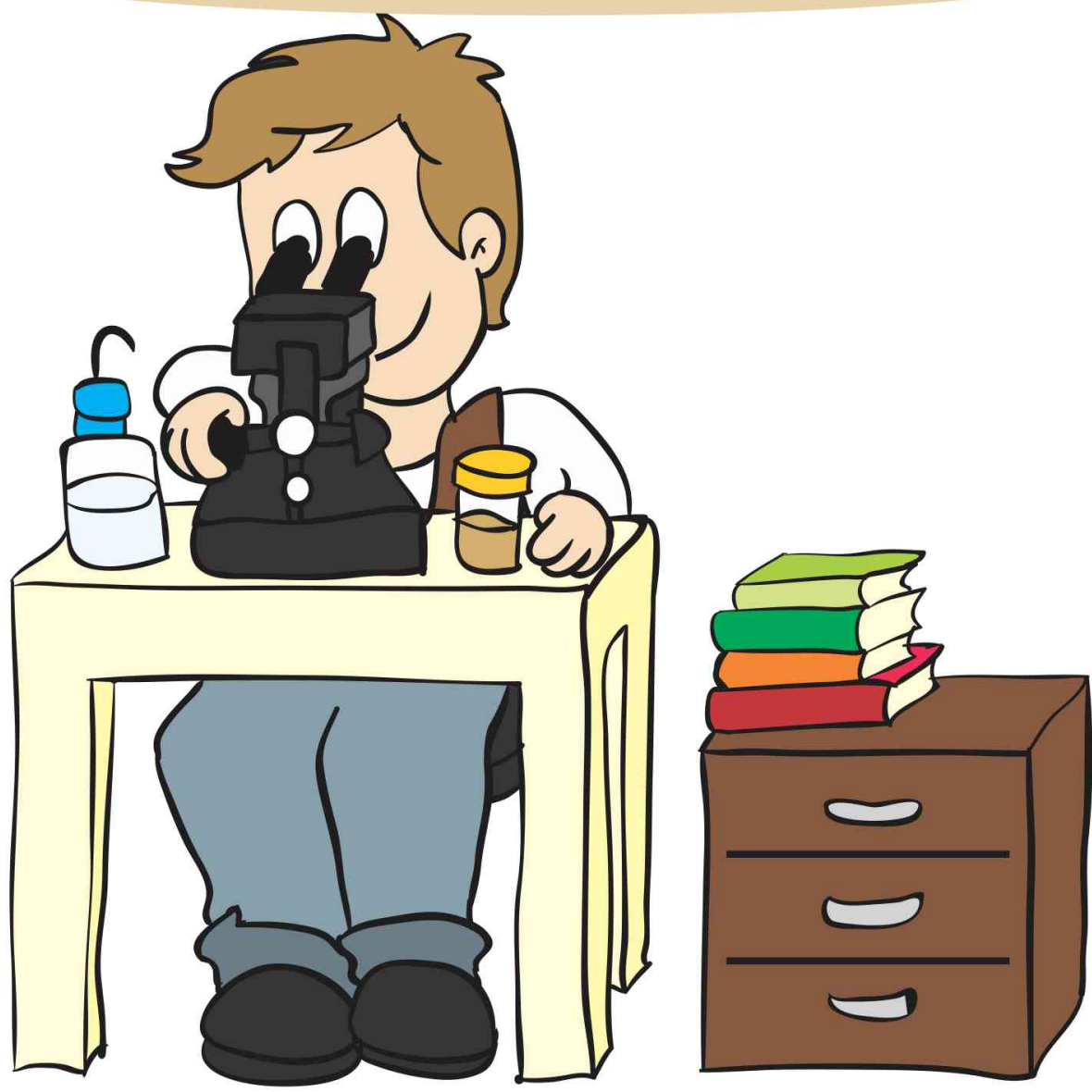


Dans l'étape suivante, les informations obtenues sont organisées et reliées aux connaissances précédentes.

Ainsi les chercheurs remarquent quelles sont les découvertes les plus importantes et qui peuvent être approuvées et divulguées.

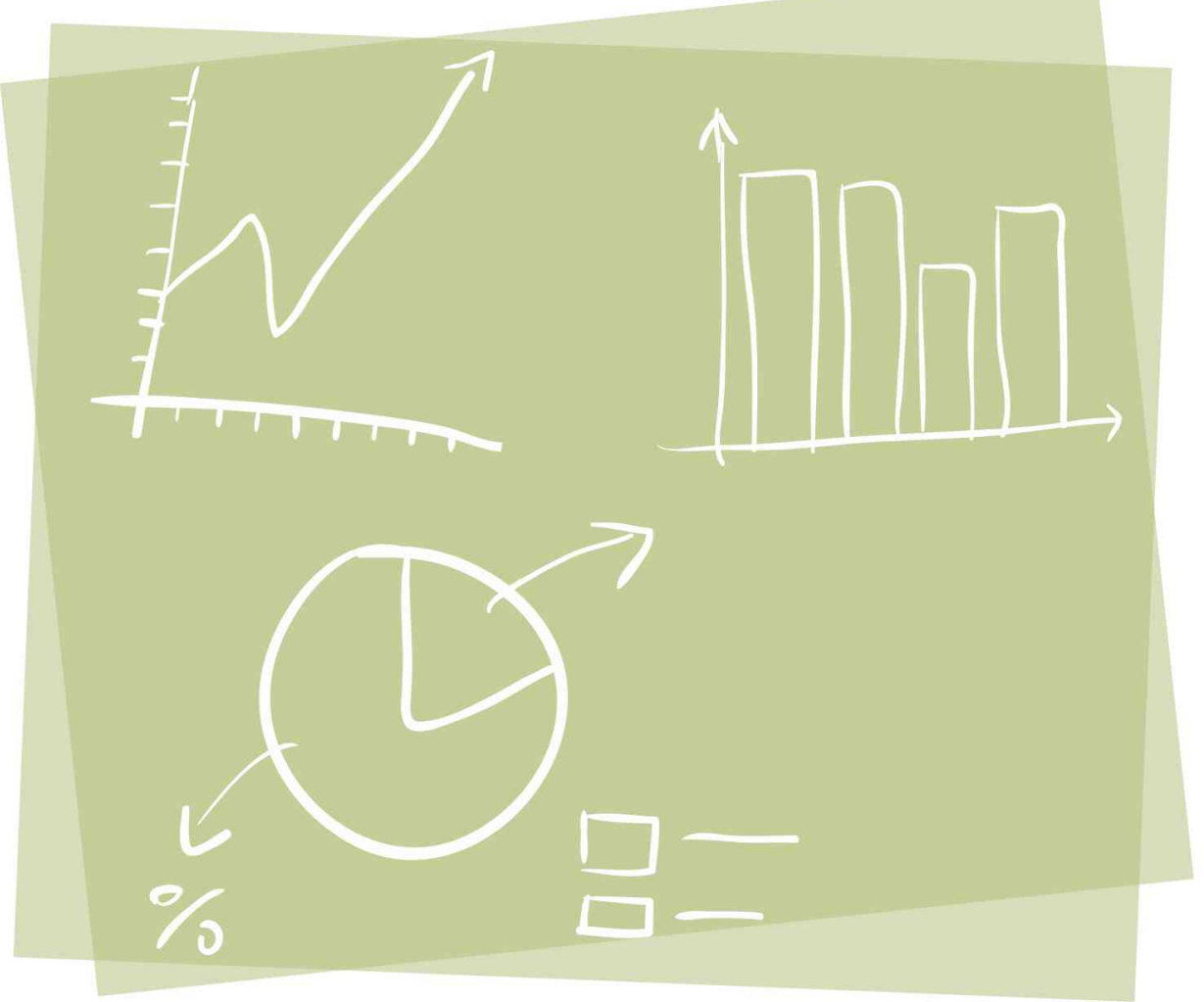

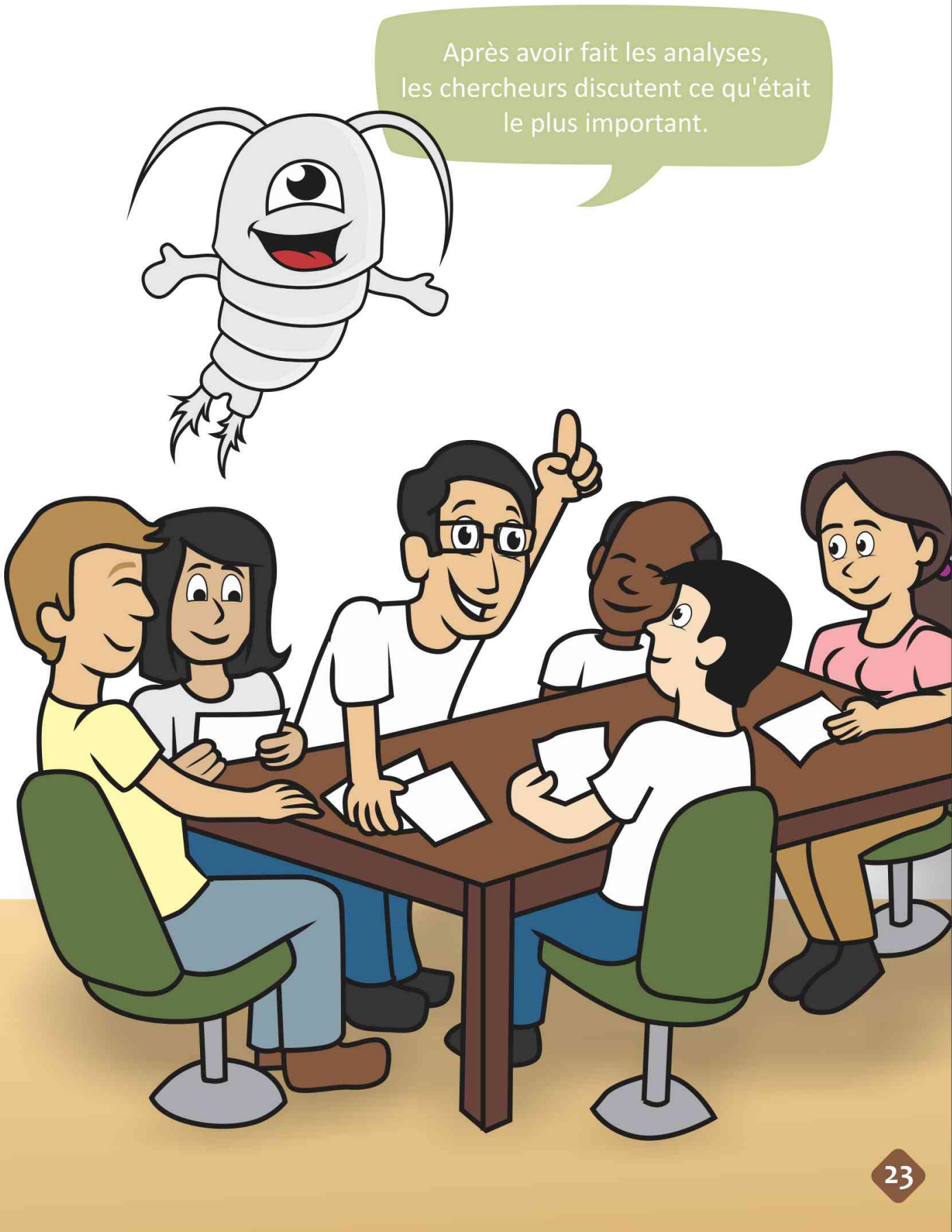


À la fin, les chercheurs publient leurs découvertes par divers moyens. La divulgation des résultats de la recherche doit être faite. C'est seulement ainsi que la société et les autres chercheurs peuvent savoir ce que a été réalisé et découvert. Ces publications peuvent être utilisées dans les nouvelles activités scientifiques, ainsi que pour une meilleure compréhension et usage de l'environnement. Les fruits de la science peuvent être apportés aux publics différents à travers des dépliants, livres, revues, documentaires, articles scientifiques, etc.

\section{REMIERCIEMIENTS}

Nous saluons le partenariat qui incluait au sein de notre projet le personnel, les laboratoires et l'équipement des institutions de l'enseignement et de la recherche: UEMG, UNESP, UnB, UCB, HidroEX et, particulièrement I'Université de Sao Paulo (USP), surtout le coordinateur du projet, Prof. Dr. Carlos Eduardo Falavigna da Rocha. La coopération interinstitutionnelle est notre objectif. A la fin, nous devons accentuer I'indispensable appui financier du FAPs (manufacture d'équipement et des pièces mécaniques), mais aussi de CNPq - Conseil National du Développement Scientifique et Technologique) et de FAPESP - Fondation pour l'Appui de Recherche de l'État de São Paulo. C'est le financement de la recherche qui garantit le progrès et la sustentation de l'avancement technique d'un pays.

Nous remercions vivement les personnes qui vivent et travaillent aux sites étudiés pour leur acceuil: les collectivités locales, les guides touristiques et le personnel des unités de conservation. Sans eux cette recherchen'aurait pas été possible.

\section{CONTACTS}

\section{Responsable du projet:}

Carlos Rocha

cefrocha@ib.usp.br

\section{llustrations:}

VictorMachado

victorilustracao@gmail.com

www.victorilustrador.com.br
Bon, mes amis, je dois partir! J'espère que vous avez aimé mon monde et que maintenant vous regarderez vos rivières, lacs et sources avec d'autres yeux. Et il va de soi, que vous êtes toujours bienvenus à participer avec nous en suivant les nouveautés du projet sur notre blog!

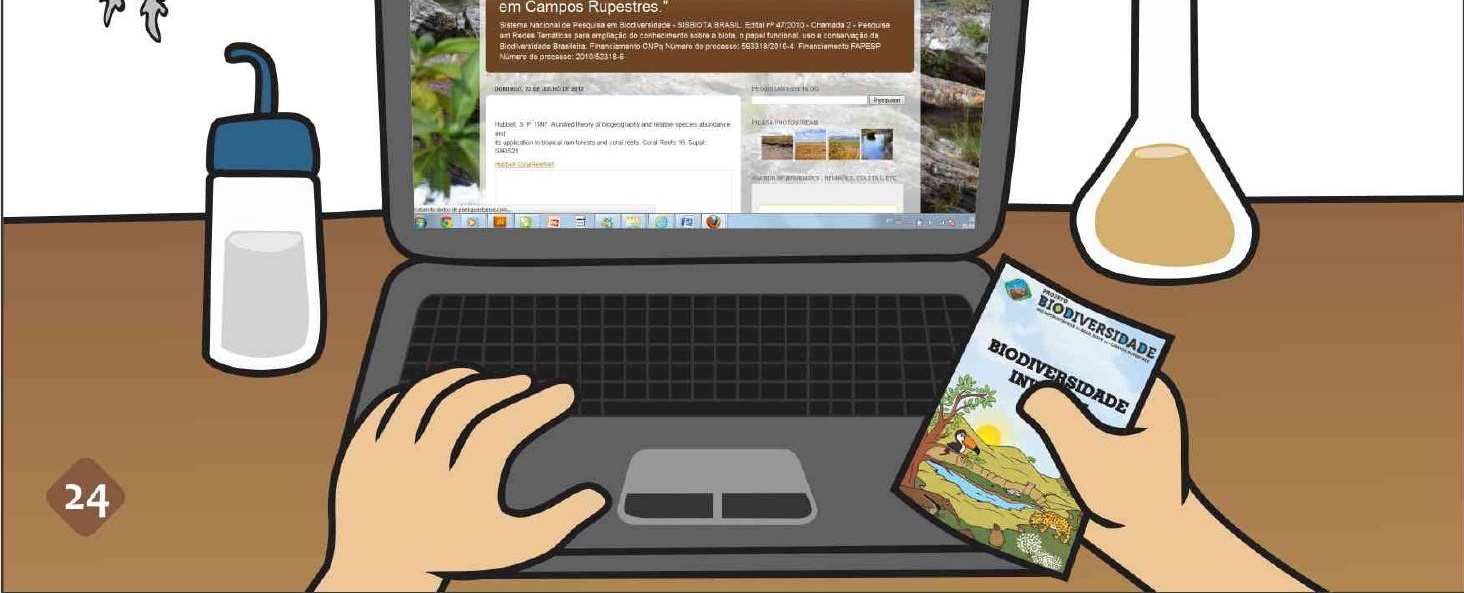




\section{PARTICIPANTS AU PROJET}

\section{Chercheurs}

Institution

Dr. Carlos Eduardo Flavigna da Rocha

USP

Dra. Cristiane Freitas de Azevedo Barros

UEMG

Dr. Daniel Previattelli

USP

Dr. Edinaldo Nelson dos Santos-Silva

INPA

Dra. Eliana Aparecida Panarelli

UEMG

Dr. Gilmar Perbiche-Neves

USP

Dra. Lourdes Maria Abdu El-moor Loureiro - UCB

Dr. Paulo Henrique Costa Corgosinho

UNESCO/IHE

Dr. Ricardo Lourenço Pinto

UnB

\section{Assistance Technique}

Elise Vargas Pereira

USP

Victor Machado Martins

UEMG

Ana Brancelj

\section{Initiation Scientifique}

Angela Miazaki UEMG
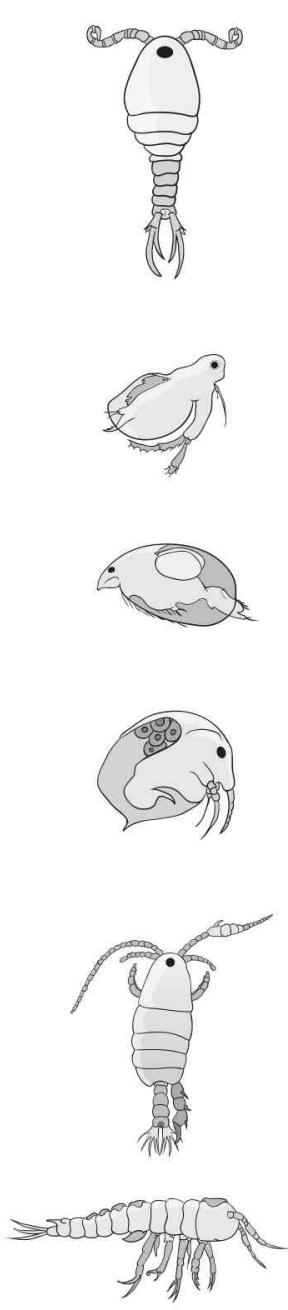

Financiament CNPq: 563318/2010-4. Financiament FAPESP: 2010/52318-6
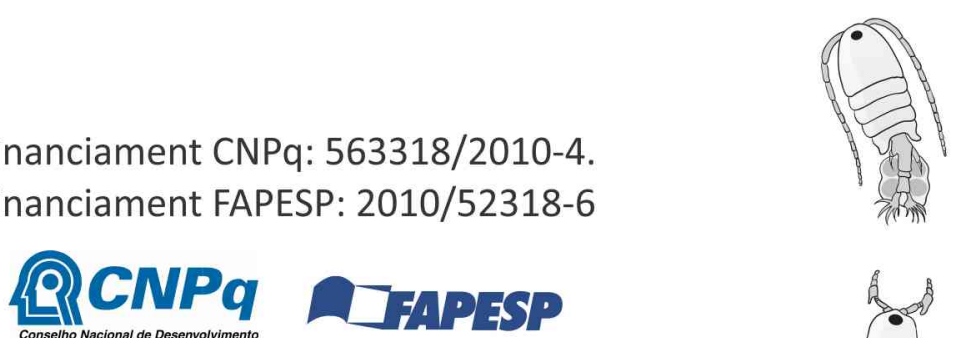
Q TAPESP 